\title{
Active limited-angle tomographic phase microscope
}

Arkadiusz Kuś

Wojciech Krauze

Małgorzata Kujawińska

\section{SPIE.}




\title{
Active limited-angle tomographic phase microscope
}

\author{
Arkadiusz Kuś, ${ }^{*}$ Wojciech Krauze, and Małgorzata Kujawińska \\ Warsaw University of Technology, Institute of Micromechanics and Photonics, Faculty of Mechatronics, Św. A. Boboli 8 Street, \\ 02-525 Warsaw, Poland
}

\begin{abstract}
We demonstrate an active, holographic tomography system, working with limited angle of projections, realized by optical-only, diffraction-based beam steering. The system created for this purpose is a MachZehnder interferometer modified to serve as a digital holographic microscope with a high numerical aperture illumination module and a spatial light modulator (SLM). Such a solution is fast and robust. Apart from providing an elegant solution to viewing angle shifting, it also adds new capabilities of the holographic microscope system. SLM, being an active optical element, allows wavefront correction in order to improve measurement accuracy. Integrated phase data captured with different illumination scenarios within a highly limited angular range are processed by a new tomographic reconstruction algorithm based on the compressed sensing technique: total variation minimization, which is applied here to reconstruct nonpiecewise constant samples. Finally, the accuracy of full measurement and the proposed processing path is tested for a calibrated three-dimensional micro-object as well as a biological object-C2C12 myoblast cell. (๑) 2015 Society of Photo-Optical Instrumentation Engineers (SPIE) [DOI: 10.1117/1.JBO.20.11.111216]
\end{abstract}

Keywords: digital holography; quantitative phase imaging; holographic tomography; adaptive optics; wavefront correction; tomographic reconstruction; compressive sensing.

Paper 150166SSPRR received Mar. 15, 2015; accepted for publication Aug. 7, 2015; published online Sep. $11,2015$.

\section{Introduction}

For at least a decade, quantitative phase imaging (QPI) has been a rapidly developing field of research, in which the metrological approach is introduced into biology ${ }^{1}$ as a new standard in cell studies. When it comes to setting standards, the fact that phase information has become quantitative instead of qualitative transforms observation into measurements. However, the nature of the information obtained is not the only reason to use the QPI techniques. It is the label-free, noninvasive, rapid, and high-resolution measurement capability provided by these methods $^{2}$ that significantly contributes to the growing popularity of QPI in biology. The hot topics and challenges in modern cell biology cover such areas as cell life cycle, three-dimensional (3-D) position tracking, cell morphology and pathology, or even drug treatment of cancer cells. Each of the aforementioned research areas can benefit greatly from QPI and measurements, especially if the result was a 3-D refractive index distribution. Of course, the possible areas of interest for QPI can be further extended for example to cell pulsation ${ }^{3}$ and the more complete information on the phase of cells could also allow exploring cell adhesion and motility. ${ }^{4,5}$

There are a number of QPI techniques that could be used in order to investigate the biological objects in two-dimensions (2-D) as well as in 3-D. One method is the transport of intensity equation (TIE), which theoretically requires at least two images to reconstruct the phase and does not require coherent illumination. ${ }^{6}$ The fastest version of this method is the single-shot approach that can be performed by using chromatic aberration. ${ }^{7}$ This technique provides the minimum number of intensities to retrieve the phase with TIE. The acquisition of the intensities is not based on mechanical movements between planes nor does it require additional time for capturing many intensity images. Still, in some cases, more planes are obtained to increase the measurement accuracy. ${ }^{8}$ This of course increases the time of the TIE measurement. Moreover, it is not sufficient to gather more intensities and also the location of the planes should be carefully specified. ${ }^{9}$ Another interesting QPI technique providing high quality and contrast results with a limited amount of noise is ptychography. ${ }^{10}$ This method does not require a complicated optical setup; however, it does require a motorized sample stage in order to scan over the sample area, which for certain applications may not be convenient. Recently, a new approach, in which moving components were replaced with coded illumination, has been presented. ${ }^{11-13}$ Another very interesting technique in the QPI group is spatial light interference microscopy, ${ }^{14}$ which, if modified, is capable of 3-D tomographic reconstruction and is then called spatial light interference tomography (SLIT). ${ }^{15,16}$ These techniques are particularly interesting with respect to the methods described in this paper. In terms of the crucial optical components in both approaches, a spatial light modulator (SLM) and a high numerical aperture (NA) microscope objective are used. However, the SLIT tomographic reconstruction is performed in a completely different manner than in tomographic phase microscopy. The SLIT technique requires axial instead of angular scanning of the sample. Even though this is a simple operation using a motorized stage of a microscope, it is still mechanical movement and often high accuracy of the sample position is important (up to tens of nanometers). In the case of tomographic phase microscopy, as presented in this paper, mechanical scanning can be avoided in order to obtain various directions of illumination of the sample. One of the benefits of SLIT is the fact that this method 
uses incoherent light and therefore is not affected by the speckle noise.

The method used in this paper is based on digital holographic microscopy (DHM), ${ }^{17}$ which uses interference to reconstruct the complex optical field in the sample plane. The setup for this technique may be using an object and reference beam, which is considered as a complication and a source of system instability. In principle, this may not always be an issue, since it is possible to overcome this with a common path ${ }^{18,19}$ or shearing ${ }^{20}$ configuration. A certain benefit of DHM is the capability of a single-shot measurement and the freedom of refocusing and propagating the complex optical field to different planes. However, this technique still provides only 2-D information on an integrated phase of the measured object. Furthermore, in digital holography the axial resolution is much lower than lateral. These issues may be resolved with tomographic reconstruction. In this technique, a set of holograms acquired at different viewing angles is recorded. Then, either phase or amplitude of the complex optical field, retrieved from a set of off-axis holograms, is processed with a tomographic reconstruction algorithm. This may be realized within 3-D Fourier domain, in which the Fourier transforms of the calculated images are assembled based on projection or diffraction approach. ${ }^{21}$ An alternative realization of the projection approach is to stack the phase or amplitude images and create a sinogram of the object, which is related to the 3-D reconstruction by inverse Radon transform, ${ }^{22}$ being adopted from computed tomography. The alternative realization of the sinogram-based diffraction approach requires the complex optical field propagated to the center of the object as the input. The only possible method of performing a fast measurement for holographic tomography is to illuminate the sample from different directions without rotating the object. However, in some cases, such as red blood cells membrane fluctuations analysis, the speed of the tomographic measurement may be insufficient. In this case, still, the basic DHM technique using a single projection could be used to provide a 2-D image. ${ }^{23}$ Recent research has already covered different configurations for 3-D reconstruction of refractive index or complex optical field ${ }^{21,24-35}$ as well as amplitude for fluorescent response. ${ }^{36}$ In these systems, the illumination direction is always altered mechanically using a motorized tilting mirror mounted on a stepper motor ${ }^{24-29}$ or in a much faster version using galvanometer mirrors. ${ }^{21,30-33}$ Alternatively, there has been a solution where the beam was directed using a rotating prism ${ }^{35}$ and a solution, in which a single mirror from a digital micromirror device acted as a point source. ${ }^{36}$ These solutions lead to a projection set limited by the aperture of the illumination and imaging systems; this cannot be avoided in fast tomography systems, and is also the case in the system presented in this paper. However, we propose a robust and diffraction-based method for altering the illumination angle in the limited-angle holographic tomography. Our idea of scanning is vibration-free, offers convenient point-to-point control of the illumination angle, high stability of the selected angle of illumination, and is also faster than the systems in which a mirror is mounted on a stepper motor. Additionally, it is possible to correct the wavefront error present in the specimen plane in order to provide the best quality reconstruction. Wavefront error would appear in a galvanometer-mirror-based system due to lower quality of the scanning mirrors caused by the thickness of the substrate in order to satisfy the moment of inertia requirements of the rotating mirror. Applying standard reconstruction algorithms to the data acquired with limited NA of the optical system leads to the degradation of the final result of the measurement. This is the effect of the missing information region and is especially visible in the direction of the optical axis of the measurement system. ${ }^{37,38}$ Therefore, we also developed a modified tomographic algorithm based on the total variation minimization (TVM): total variation iterative constraint (TVIC) method, which improves both external geometry and phase distribution reconstruction in the direction of optical axis as well as in the perpendicular direction.

\section{Materials and Methods}

\subsection{Experimental Setup}

The tomographic microscope presented in this paper is based on a vertical setup of a holographic microscope for transparent objects in Mach-Zehnder configuration. ${ }^{39}$ This configuration is well suited for biological samples, which are usually in a liquid environment. The crucial modification of the holographic microscope is the introduction of a reflective phase-only SLM in the beam, which illuminates the object. Moreover, the system is designed in a way that additional modifications would enable integration into a DHM in the future. The scheme of the optical system of the holographic microscope is shown in Fig. 1.

The He-Ne laser beam $(\lambda=632.8 \mathrm{~nm})$ is delivered to the setup using a polarization-maintaining, single-mode fiber and is then collimated with a $100-\mathrm{mm}$ focal length lens. The polarizer and half-wave plate (multiorder $\lambda / 2$ plate, designed for $\lambda=633 \mathrm{~nm}$ ) are used to control the intensity and the azimuth of polarization required for the phase-only liquid crystal (LC) SLM to work with. Then, the beam is split into reference and object beams by a 50:50 beam-splitting cube. The reference beam is directed by the M2 mirror toward the CCD. It passes through a beam expander, which consists of $\mathrm{L} 3\left(f^{\prime}=30 \mathrm{~mm}\right)$

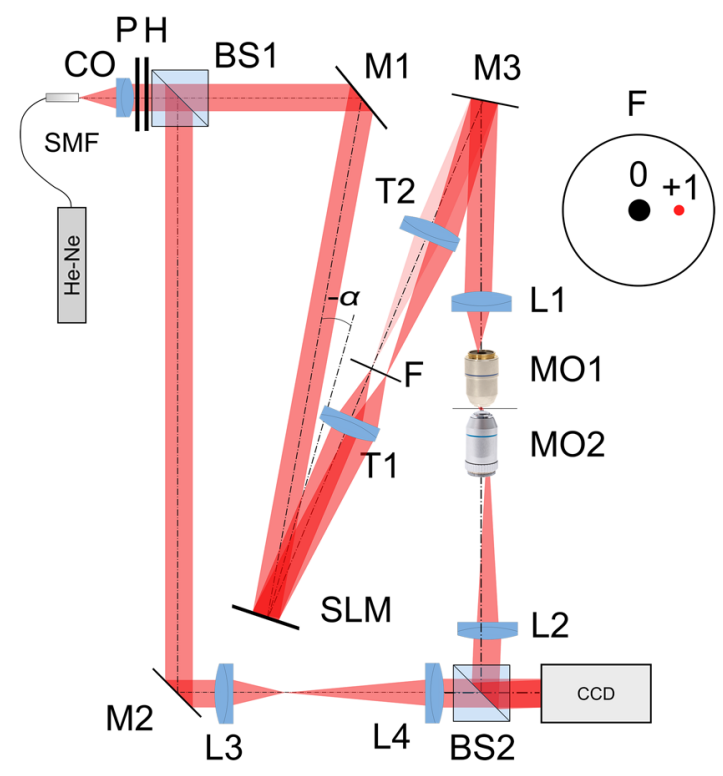

Fig. 1 Tomographic microscope setup. He-Ne: laser; SMF: singlemode fiber; $\mathrm{CO}$ : collimating objective; $f^{\prime}=100 \mathrm{~mm}$; $\mathrm{P}$ : linear polarizer; $\mathrm{H}$ : half-wave plate; BS1 and BS2: beam splitter cube; M1, M2, and M3: flat mirror; SLM: spatial light modulator; T1, T2, L1, L2, L3, and L4: lens; F: spatial filter; MO1: 100× microscope objective; MO2: $40 \times$ microscope objective; and CCD: charge coupled device camera. 

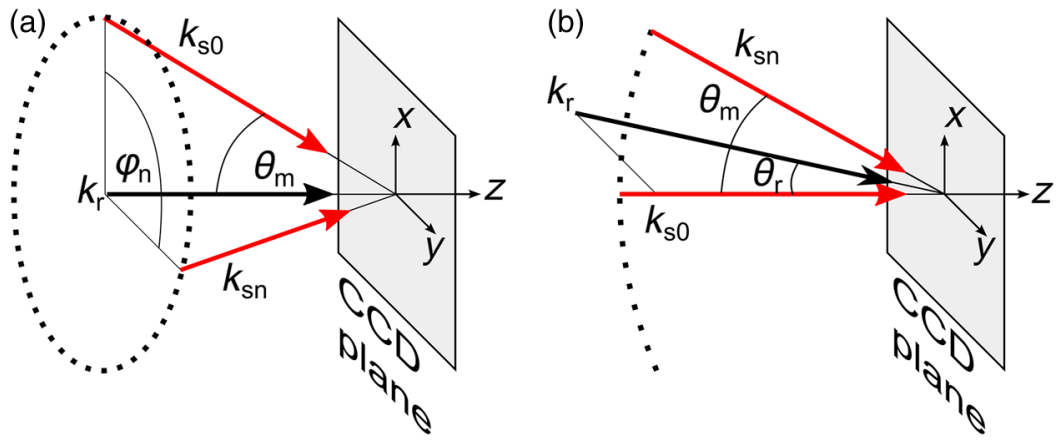

Fig. 2 Basic illumination scenarios in tomographic measurement presented in the image plane, (a) the result of circular scanning of the back focal plane (BFPL) of the MO1 microscope objective with object beam, $k_{\mathrm{sn}}$ : $n$ 'th sample wave vector, $n=1$ to total number of projections, $k_{\mathrm{r}}$ : reference beam, $\theta_{\mathrm{m}}$ : maximum sample beam incidence angle in the image plane, $\varphi_{n}$ : azimuth of the $n^{\prime}$ th projection, (b) the result linear scanning of the BFPL of the MO1 objective, $\theta_{\mathrm{r}}$ : reference beam angle of incidence in the camera plane.

and $\mathrm{L} 4\left(f^{\prime}=150 \mathrm{~mm}\right)$. This component is useful for introducing a spatial carrier in the holograms. The object beam is directed through the M1 mirror toward the high-resolution $(1920 \times 1080$ pixels, $8.0 \mu \mathrm{m}$ pixel pitch, $93 \%$ fill factor $)$ liquid crystal on silicon (LCoS) SLM, PLUTO-VIS-014 (Holoeye Photonics AG, Germany). The beam impinges on the SLM at the angle $-\alpha$ equal to $8 \mathrm{deg}$. The SLM is placed in the front focal plane (FFPL) of the T1 lens $\left(f^{\prime}=100 \mathrm{~mm}\right)$. The T2 lens $\left(f^{\prime}=75 \mathrm{~mm}\right)$ acts as a second lens of the (T1-T2) telescope system. The spatial filter $F$, which is used to block zero diffraction order and reflection from the SLM cover glass, is optional. The image of the SLM surface is created in the back focal plane (BFPL) of the L1 lens $\left(f^{\prime}=250 \mathrm{~mm}\right)$. The beam is focused in the FFPL of the MO1 microscope objective (Olympus UPlanFLN $100 \times / 1.3$, infinity-corrected, $0.17 \mathrm{CS}$ - cover slip, WD $=0.2 \mathrm{~mm}$ ). If a blazed grating is displayed on the modulator, the incident plane wave is diffracted into a single diffraction order. Expanded by the telescope, the diffracted beam is focused in the FFPL of the MO1 objective and creates a tilted illumination of the sample. When a series of blazed gratings with controlled frequency and direction is displayed on the modulator, the FFPL of the MO1 is scanned (see Sec. 3). Each point in the FFPL corresponds to an inclined beam in the specimen plane. Imaging of the sample illuminated in this manner is performed using the MO2 microscope objective (Leica HCX Pl APO $40 \times / 1.25$ to $0.75 \mathrm{CS}, \mathrm{WD}=0.1 \mathrm{~mm}$ ). The plane wave, illuminating the sample, is focused in the BFPL of the MO2 and collimated using the L2 tube lens $\left(f^{\prime}=200 \mathrm{~mm}\right)$. The CCD camera (JAI BM-500GE, $2456 \times$ 2058 pixels, $3.45 \mu \mathrm{m}$ pixel size) is placed in the BFPL of the L2 lens. The magnification $M$ of the imaging part of the system is 38 .

In this tomographic microscope, the SLM replaces two rotary mirrors, which would normally be necessary to scan the aperture of the MO1 microscope objective. Implementing SLM as an active element for scanning directions of the object-illuminating beam provides the freedom of different illumination scenarios. Below, we describe two basic types of illumination scenarios: linear and circular. In the case presented in Fig. 2(a), a spatial filter $F$ (Fig. 1) is placed in the Fourier plane of the T1 lens. Scanning the aperture of the MO1 along a circle results in illumination beams lying on a cone. In this approach, the frequency of the spatial carrier rotating in the camera plane is constant for each projection, which is beneficial for hologram processing. In the scanning method presented in Fig. 2(b), the illumination beams lie in one plane. This concept allows capturing an image for normal illumination. Here, the spatial carrier frequency is no longer constant, which might lead to issues with too low or too high fringe density.

The results of data acquisition are presented in Fig. 3. The spatial carrier frequency in Figs. 3(a) and 3(b) depends on the $\theta_{\mathrm{m}}$ illumination angle only and for Fig. 3(c) can also be adjusted with $\theta_{\mathrm{r}}$ angle (with a single, mechanical tilt of the M2 mirror) to obtain optimal conditions for phase reconstruction for every projection. The spatial carrier frequency should not exceed the Nyquist limit for $\pm \theta_{\mathrm{m}}$. (a)

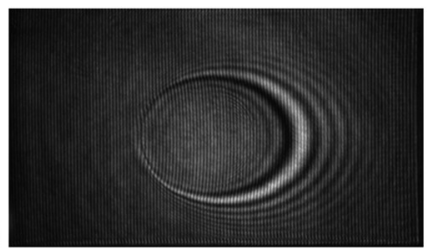

(b)

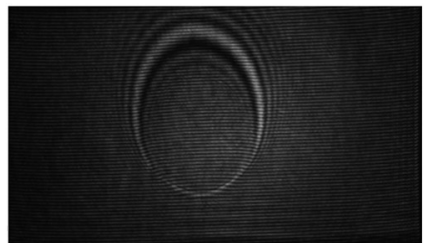

(c)

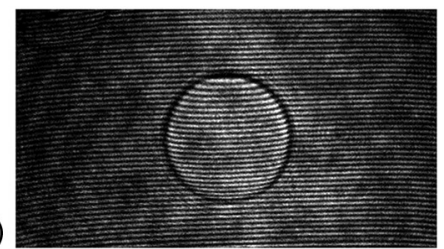

Fig. 3 Off-axis holograms of a $23.5 \mu \mathrm{m}$ PMMA microsphere: (a) circular scanning measurement (CSM) scenario, angle of incidence of the illumination beam in the specimen plane $\theta_{\text {in }}=53.5 \mathrm{deg}, \theta_{\mathrm{r}}=0 \mathrm{deg}$, $\varphi_{\mathrm{n}}=0 \mathrm{deg}$, (b) CSM scenario, angle of incidence of the illumination beam in the specimen plane $\theta_{\text {in }}=53.5 \mathrm{deg}, \theta_{\mathrm{r}}=0 \mathrm{deg}, \varphi_{\mathrm{n}}=270 \mathrm{deg}$, and (c) linear scanning measurement (LSM) scenario, $\theta_{\text {in }}=0$ deg. 


\subsection{Spatial Light Modulator as an Active Device in Tomographic Microscope}

\subsubsection{Beam steering}

The main function of the SLM in the tomographic microscope is a vibration-free, diffraction-based beam deflection, which is used to acquire a set of holograms for a series of illumination angles. For this purpose, a series of blazed gratings is displayed on the SLM. For the circular illumination scenario, the blazed grating is rotated in the SLM plane; for the linear scenario, the period of the grating is varied for each projection. However, several conditions and limitations of SLMs need to be considered and taken into account to obtain illumination distribution that would allow a high-quality tomographic reconstruction. Apart from the accuracy and the quality of the holograms, the quality of the reconstruction depends strongly on the angular span of the projections, which should be as large as possible, and the number of the projections, which should also be maximized. Unfortunately, the SLM can only display $N$ blaze steps with width equal to $N$-times pixel size $(8 \mu \mathrm{m})$, which limits the period of the displayed grating and maximum diffraction angle to $2.27 \mathrm{deg}(N=2)$. However, should the measurement system have optimum energy efficiency, the minimum period of a blazed grating is further limited to $N=8$ (diffraction efficiency $\eta=0.95$ for the -1 st order $^{40}$ ). Thus, the smallest period of the blazed grating displayed on the SLM should be equal to $64 \mu \mathrm{m}$, which corresponds to $0.57 \mathrm{deg}$ of the diffraction angle. On the other hand, the maximum possible illumination angle is in this case limited by the NA of the MO2 objective and the refractive index of the immersion medium $(n=1.4878)$, which means that $\theta_{\max }=57.2 \mathrm{deg}$. This imposes the minimal angular magnification of the T1-T2-L1-MO1 part of the system.

However, even if this is taken into account, the angle of illumination in the sample plane is not linearly dependent on the blaze width. This means that in the systems, which have angular magnification of the telescopes around $100 \times$, near the $\theta_{\max }$, a sample can be illuminated every $4 \mathrm{deg}$ and the cut-off illumination angle is $1 \mathrm{deg}$. The normal illumination is realized when no grating is displayed on the modulator. It is possible to increase linearity and decrease angular step near the maximum illumination angle if the angular magnification $(M \alpha)$ of the telescopes is increased. In our system, we propose the $M \alpha=240$. The minimum blazed grating period used is 15 pixels, which corresponds to $\theta_{\text {in }}=53.5 \mathrm{deg}$ incidence angle in the sample plane. In a linear scanning scenario, the cut-off illumination angle is $1 \mathrm{deg}$ and the angular step near $\theta_{\text {in }}$ is equal to $2 \mathrm{deg}$. This nonuniform distribution will be taken into account in the tomographic reconstruction. This is not a problem with circular scanning, which is based on rotating a 15 pixel blazed grating at $1 \mathrm{deg}$ interval. In the linear scanning scenario, there were 265 holograms acquired with angular step of 0.2 to $2 \mathrm{deg}$. For the circular scanning scenario, there were 360 holograms captured at one degree of rotation intervals, at $\theta_{\text {in }}=53.5 \mathrm{deg}$.

Finally, the measurement speed with an SLM is limited by the refresh rate of the phase microdisplay, which is $60 \mathrm{~Hz}$. However, in order to avoid display errors, the maximum achievable frame rate of the display would have to be limited at least by half (toggle rate). In this particular case, the displaying rate was limited by the software efficiency (Java full-screen display procedures run by MATLAB) to $11 \mathrm{~Hz}$. Currently, the optical beam steering does not match the performance of galvanometermirror-based systems, in which the top speed of data acquisition is 150 times higher, ${ }^{31}$ however, it is possible to increase the speed of the system using a $500 \mathrm{~Hz}$ SLM.

\subsubsection{Wavefront correction}

SLMs are common tools in wavefront correction ${ }^{41,42}$ and are also used in microscopy to improve image quality. ${ }^{43}$ Phaseonly, reflective modulators are also used in interferometry for wavefront shaping. ${ }^{44}$ In this case, the correction of the object beam wavefront compensates for the shape of the SLM, which is not perfectly flat, and the aberration that is present at angles close to $\theta_{\max }$. Also, if a biological sample was measured, the refractive index mismatch between the immersion liquid and the specimen would result in spherical aberration. In this approach, the calibration is a convenient procedure because it is only necessary to find a sample-free region and create a look-up table (LUT) once per measurement scenario and a sample with specific refractive index.

In this paper, to display a phase image for wavefront correction, Zernike orthogonal polynomials ${ }^{45}$ are used to fully characterize the aberrations. SLM is rectangular, therefore it is important that the polynomials are calculated over a rectangular aperture ${ }^{46}$ instead of a unit disk. In this work, the first 15 Noll indices of the polynomials were used. Processing the reference image to calculate correction map could of course also be realized using averaging, filtering methods such as bidimensional empirical mode decomposition ${ }^{47}$ or alternative image reconstruction technique based on orthogonal moments. ${ }^{48}$ However, the established theory for calculating the wavefront aberrations based on the Zernike polynomials of the reference measurement appears to be the most functional approach for wavefront correction.

The pixel size of the modulator is $P_{\mathrm{m}}=8 \mu \mathrm{m}$, while the camera pixel size is $P_{\mathrm{c}}=3.45 \mu \mathrm{m}$. The basic condition for reconstructing the wavefront for the SLM with a camera used for imaging is to maintain the relation $P_{\mathrm{m}}>P_{\mathrm{c}}$. In the case presented here, $P_{\mathrm{m}}$ in the camera plane is $1.62 \mu \mathrm{m}$, which means that the resolution of the SLM is not fully used in wavefront correction and additional errors may occur during the processing of the reference wavefront map.

The result of the calculation of the Zernike-polynomialsbased wavefront map for the SLM is presented in Fig. 4. Calculation of the wavefront map does not take into account the higher frequencies in the phase. In this way, the calibration is not affected by the noise introduced by the sample-free area and only corrects aberration introduced by the optical system and the shape of the modulator.

The benefit from correcting the wavefront is the fact that the quality of the reconstructed image is improved. Moreover, the sample does not need to be removed in order to place a calibration object, which is convenient. Also, when multiple objects are measured within one sample chamber, the processing of the phase images is simplified-there is no need to subtract the background wavefront or perform additional deconvolution operations.

\subsection{Measurement Procedure}

In order to perform calibration and measurement using the active tomographic microscope with optically controlled projections, a few steps must be performed. This is shown briefly in Fig. 5.

In the approach presented in this paper, the preprocessing part of the measurement is extended. The key part of the 
(a)

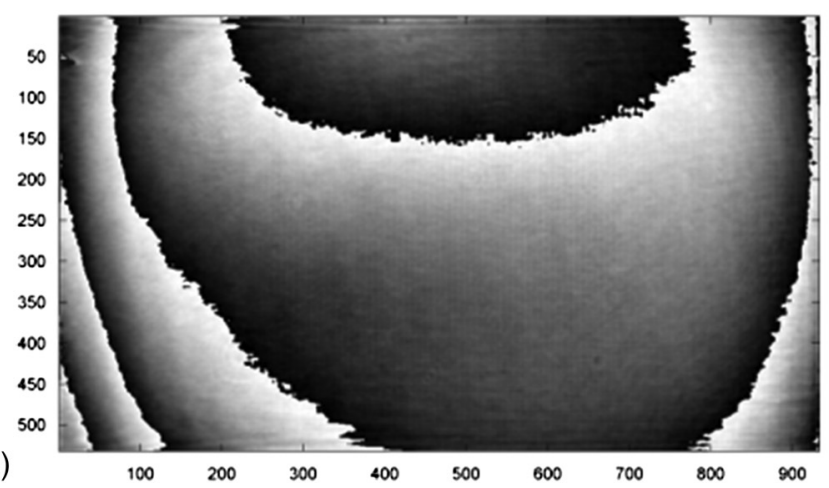

(b)

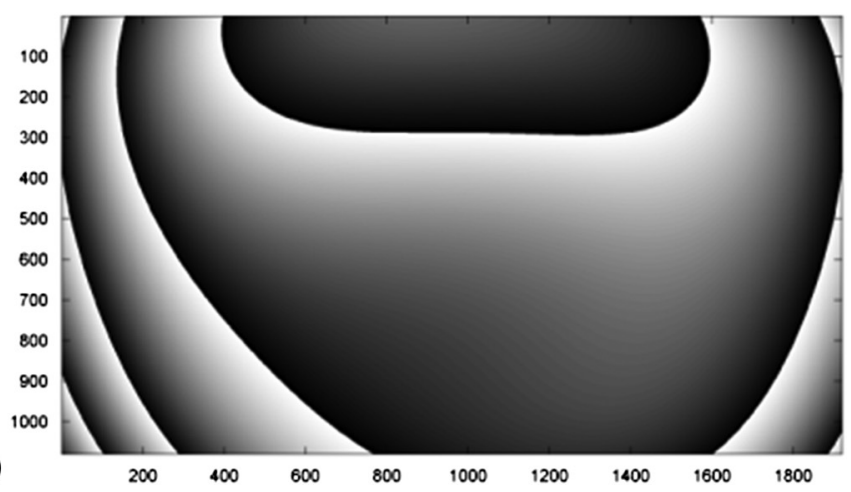

Fig. 4 Wrapped correction phase for $\theta_{\mathrm{m}}$ illumination angle: (a) the reference wavefront and (b) wavefront reconstructed from calculated Zernike polynomials for coefficients 1 to 15 th Noll index.

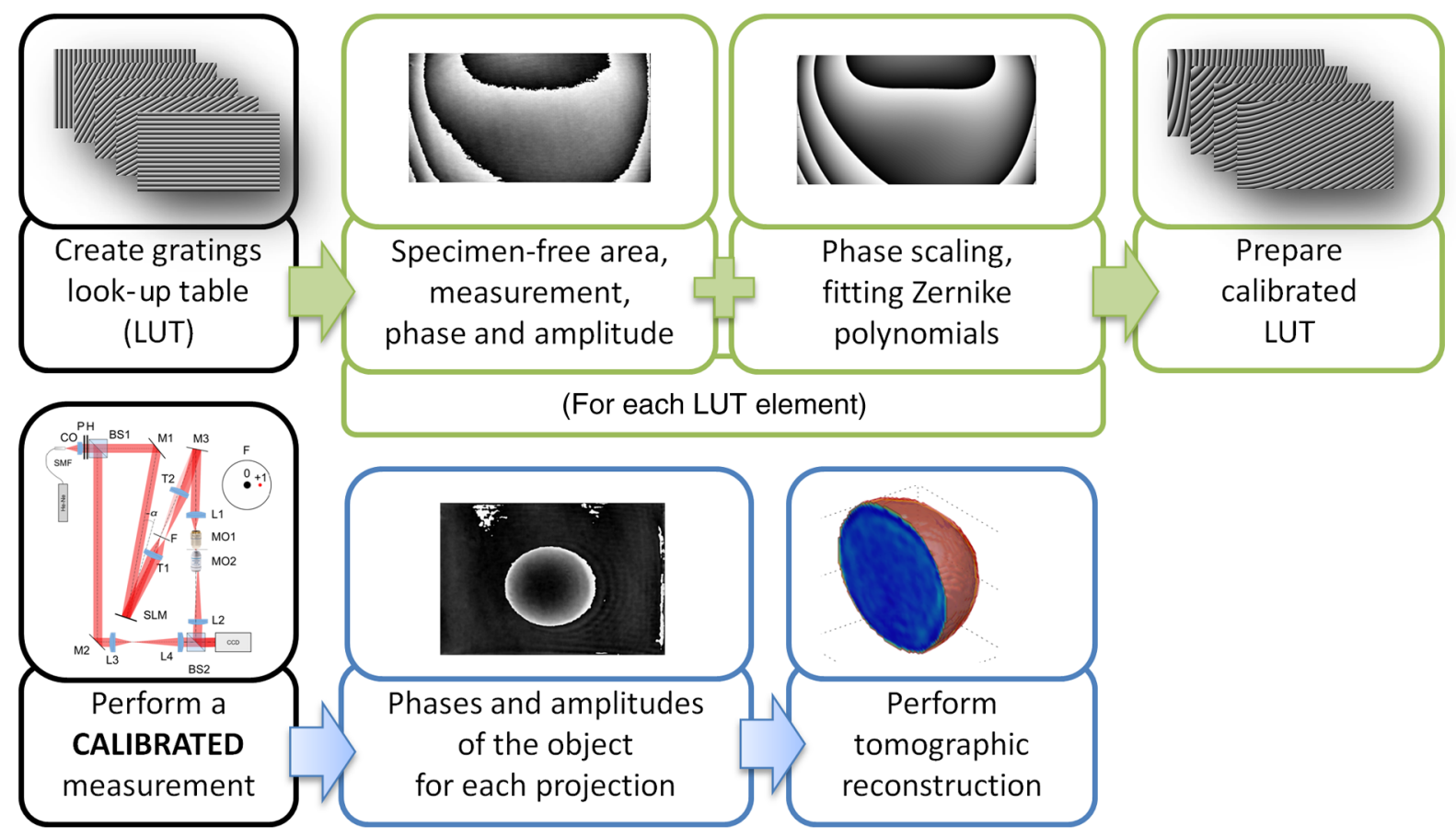

Fig. 5 Measurement procedure for look-up table (LUT)-calibrated measurement.

preprocessing is calculating Zernike polynomials followed by preparation of calibrated LUT containing a blazed grating for each projection. The effect of the superposition of the correction wavefront and the blazed grating is presented in Fig. 6. The maximum intensity of the created pattern is adjusted to match the required $2 \pi$ phase shift.

The result of implementation of the regular and corrected LUT cells is presented in Fig. 7. The wavefront has been successfully corrected over the sample area and most of the SLM. It can be noticed that errors caused by the borders of the SLM are not corrected well. In addition, apparently the correction wavefront might not fully match the real aberrated wavefront. This might occur if the SLM border is not perfectly parallel to the camera plane. For this reason, a calibration method needs to be proposed in the future. Also, placing an amplitude mask in the plane conjugate with the SLM surface would reduce the diffraction caused by the border of the modulator.

\subsection{Tomographic Reconstruction}

The illumination architectures described above do not provide data from full tomographic angular range and therefore require a special approach. According to the diffraction slice theorem, these architectures result in empty areas in the Fourier domain, ${ }^{21}$ which in turn strongly influence reconstruction resolution, which becomes anisotropic. Anisotropic resolution is not the only artifact present in the reconstruction. Another is a distorted geometry of the reconstructed object- the shape is elongated in the direction in which no projections were recorded. ${ }^{37,38}$ In general, the problem of tomographic reconstruction becomes even more problematic, which in this case means that from the same set of initial data, an almost infinitely large set of solutions with a relatively small residuum may be calculated. ${ }^{49}$ A number of algorithms that compensate for the partial lack of input data have already been developed. ${ }^{50}$ The state-of-the-art approaches utilize TVM, ${ }^{51-54}$ a technique derived from compressed sensing. 


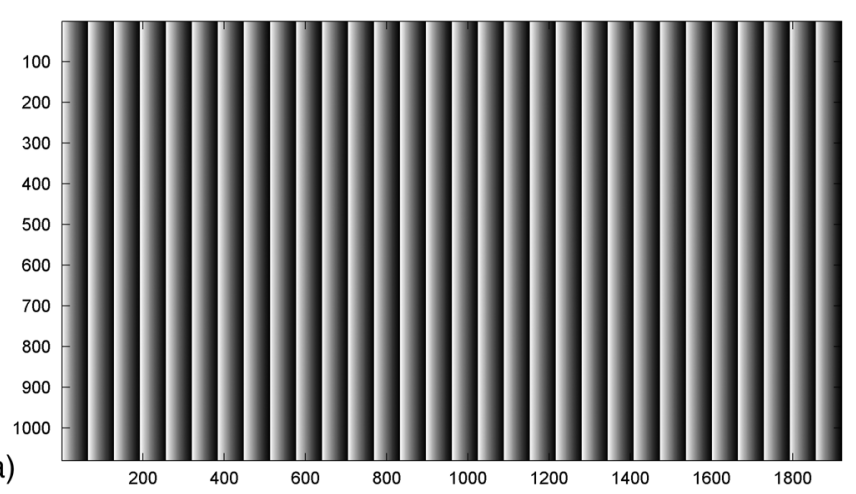

(b)

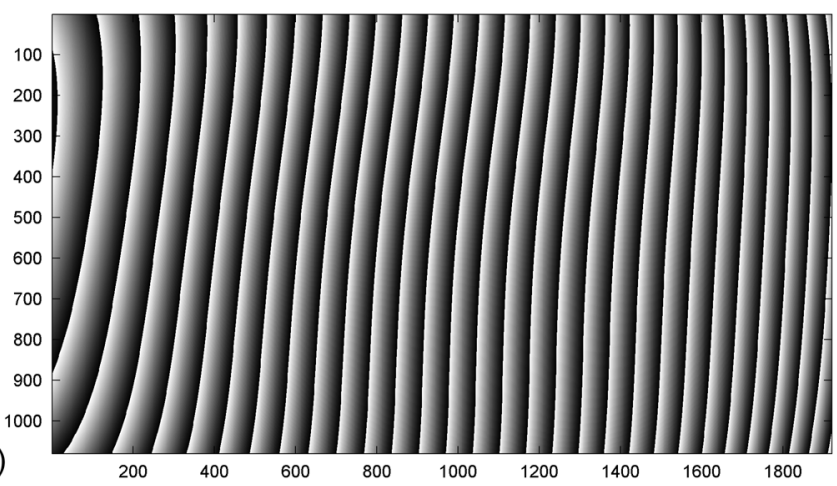

Fig. 6 An example of a LUT frame for a 36-pixel blazed grating $(\theta=28$ deg): (a) precalibration blazed grating and (b) calibrated LUT frame, modified with reference wavefront.

(a)

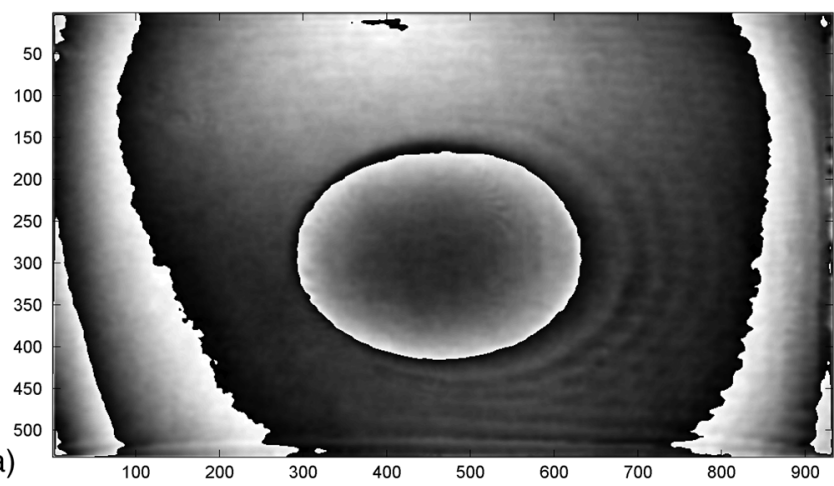

(b)

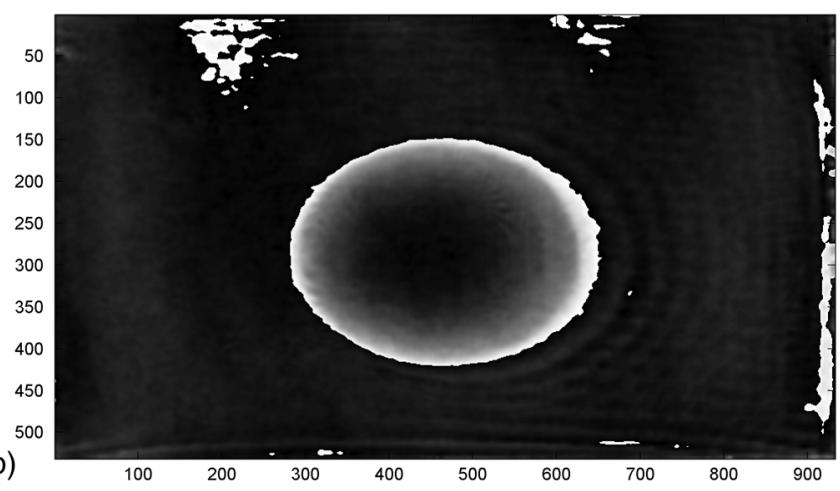

Fig. 7 Wrapped phase of a $23.5 \mu$ m PMMA microsphere: (a) measurement with a 15-pixel blazed grating and (b) measurement with a calibrated LUT frame-15-pixel blazed grating modified with a reference wavefront.

TV minimization is a very powerful regularizer that makes use of the fact that some objects' gradient is sparse. By applying the TVM iteratively in the reconstruction process, one can limit the number of possible solutions of the tomographic reconstruction, thus it is possible to retrieve full information about the object. By definition, the TVM is applicable to piecewise constant samples only. Herein, we propose a novel technique, namely the TVIC. It allows applying the TVM technique to reconstruct nonpiecewise constant samples such as biological cells. In this approach, in the first step, a piecewise constant reconstruction of the sample is calculated by means of Chambolle-Pock algorithm. ${ }^{55}$ Despite the fact that inner structures of the reconstructed sample are erroneous (by applying Chambolle-Pock algorithm we assumed piecewise constancy to a nonpiecewise constant specimen), the external geometry is retrieved correctly with very good precision. This reconstruction is binarized and treated as an external geometry mask. The knowledge about external geometry is now a powerful prior, which strongly improves the final results. In our approach, this prior is applied iteratively to the algebraic reconstruction algorithm. ${ }^{56}$ Other priors are nonnegativity constraint and smoothness of the refractive index distribution. The latter prior is implemented in the form of a 3 -D median filter of the $3 \times 3 \times 3$ size.

When algebraic reconstruction techniques are applied to 3 -D tomography, the size of the system matrix $A$ from the equation $A x=p$, in which $x$ is the reconstructed image and $p$ is the input data (sinogram), is always a problem. In our case, the system matrix $A$ has a size of approximately
$3,600,000 \times 1,000,000$, which would normally mean more than $10 \mathrm{~TB}$ of data. This is why we utilize the ASTRA Toolbox, ${ }^{57}$ which makes use of the fact that a major part of this matrix consists of zeros and so the matrix may be created with a reasonable amount of memory. It also allows the implementation of custom TV regularizers.

The algorithm results in a reconstruction with partially improved quality of inner structures of the specimen and with precisely retrieved external geometry. During the main reconstruction stage, the piecewise constancy was not assumed, making it applicable to a wide range of samples.

\subsection{Sample Preparation}

One of the two samples prepared for the experiment in this paper was a microsphere made of PMMA. The microspheres were produced by microParticles $\mathrm{GmbH}$. The diameter of the sphere was $23.5 \mu \mathrm{m}$ and the refractive index for $\lambda=632.8 \mathrm{~nm}$ is $n_{\text {sphere }}=1.489$, and it was immersed in index-matching liquid (Cargille) with refractive index $n_{\text {immersion }}=1.517$. The object was placed between two $0.17 \mathrm{~mm}$ coverslips separated with \#0 coverslip (0.08-mm thick).

As the second sample, the $\mathrm{C} 2 \mathrm{C} 12$ mouse myoblast cell line ${ }^{58}$ was prepared. The $\mathrm{C} 2 \mathrm{C} 12$ cells were maintained in DMEM high glucose medium with L-glutamine and sodium pyruvate (Gibco) supplemented with $10 \%$ fetal bovine serum (Sigma-Aldrich) and $1 \%$ penicillin/streptomycin mixture (Life Technologies) at $37^{\circ} \mathrm{C}$ in a humidified $95 \%$ air and $5 \% \mathrm{CO}_{2}$ atmosphere. Cells 
were passaged at $70 \%$ to $80 \%$ of confluence. For the experiment, cells were seeded on autoclaved $0.17 \mathrm{~mm}$ coverslips.

Cells on coverslips were rinsed briefly with cold $1 \times$ phosphate buffer saline (PBS) (Pracownia Chemii Ogólnej IIITD PAN) and fixed in 4\% paraformaldehyde (Sigma-Aldrich) for $10 \mathrm{~min}$ at room temperature. Next, the coverslips were rinsed with cold PBS buffer $(2 \times 5 \mathrm{~min}$ at room temperature $)$. Finally, the coverslips were stored in $1 \times$ PBS buffer supplemented with $0.02 \%$ sodium azide (Sigma-Aldrich). For the measurement, coverslips with cells were supported on two \#0 coverslip spacers and covered with a \#1 coverslip. For the measurement, the cells were immersed in water $\left(n_{\mathrm{w}}=1.3319\right.$ at $\lambda=632.8 \mathrm{~nm}$ and $\left.T=20^{\circ} \mathrm{C}\right)$.

\section{Results}

\subsection{Tomographic Reconstruction of a Calibrated Model}

In order to test the performance of the algorithm as well as compare the proposed scanning scenarios, a well-defined object, i.e., polymer microsphere, was measured. Two illumination architectures were used in the measurement, as described in Sec. 2.1: single-axis (linear) illumination and conical illumination. The acquired data were reconstructed with TVIC algorithm. It should be noted that the calibrated object is piecewise constant, but during the second stage of the reconstruction process piecewise constancy was not assumed. However, the piecewise constant microsphere still allows verification of the quality of the reconstruction in terms of shape and refractive index changes. Figures 8 and 9 present the reconstruction of the refractive index distribution in the microsphere based on the data captured with the single-axis illumination and the conical illumination scenarios, respectively. The color scale represents the absolute refractive index values calculated using the $a$ priori information of the refractive index of the background (certified index-matching liquid).

Figures 8 and 9 show that TVIC algorithm provides correct external geometry of the measured object. There is also visible improvement in the reconstruction of the inner structure when these results are compared with results calculated with SART+ ATV algorithm. ${ }^{26,37}$ However, it is clearly visible in Fig. 8(b) that the shape of the microsphere obtained with LSM is distorted along the direction of the optical axis, which is even more apparent in Fig. 10(a).

The asymmetry of the microsphere measured with the circular scanning scenario (along the $X$ - and $Z$-axes) is in this case less than $1.7 \%$ with a slight elongation of the reconstruction in the direction of optical axis. We consider this to be a very good result and, based on this, have decided to use only a CSM scenario. Also, the distribution of the refractive index in the $Z$ direction is much more uniform and similar to the $X$ profile for the
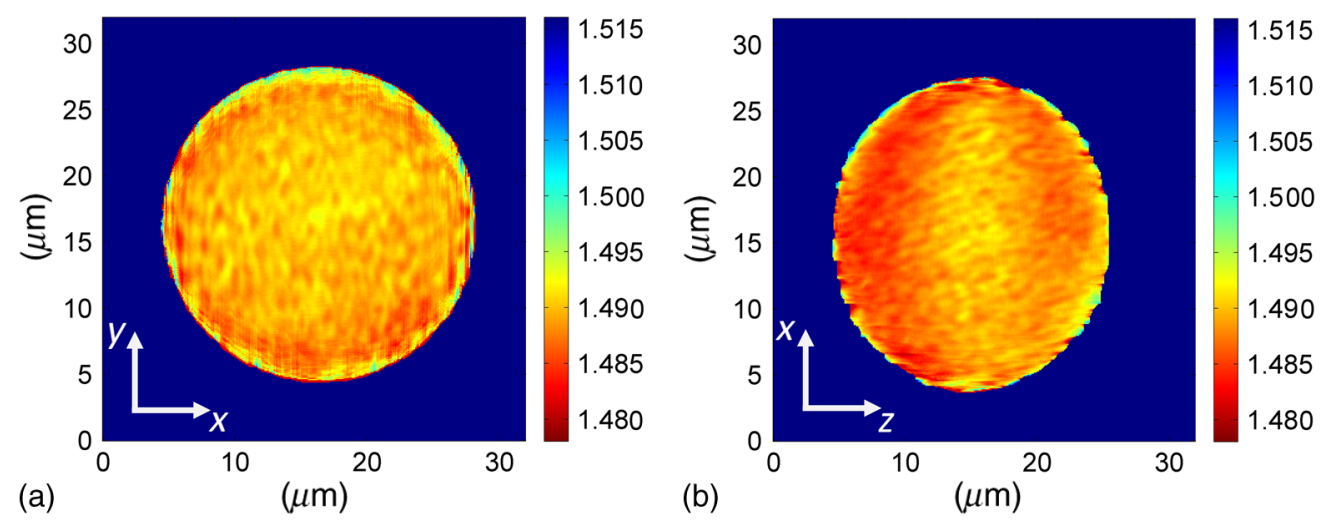

Fig. 8 Central cross section of a three-dimensional (3-D) reconstruction of the refractive index of a microsphere reconstructed from data gathered using the linear illumination scanning measurement scenario.
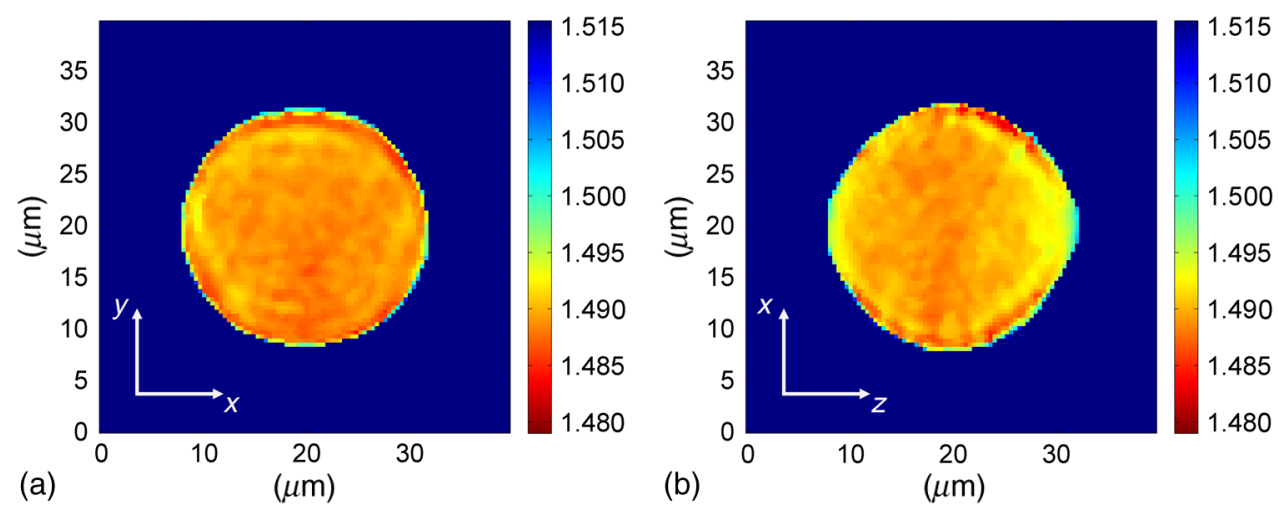

Fig. 9 Central cross section of a 3-D reconstruction of refractive index of the microsphere reconstructed from data gathered using the CSM scenario. 

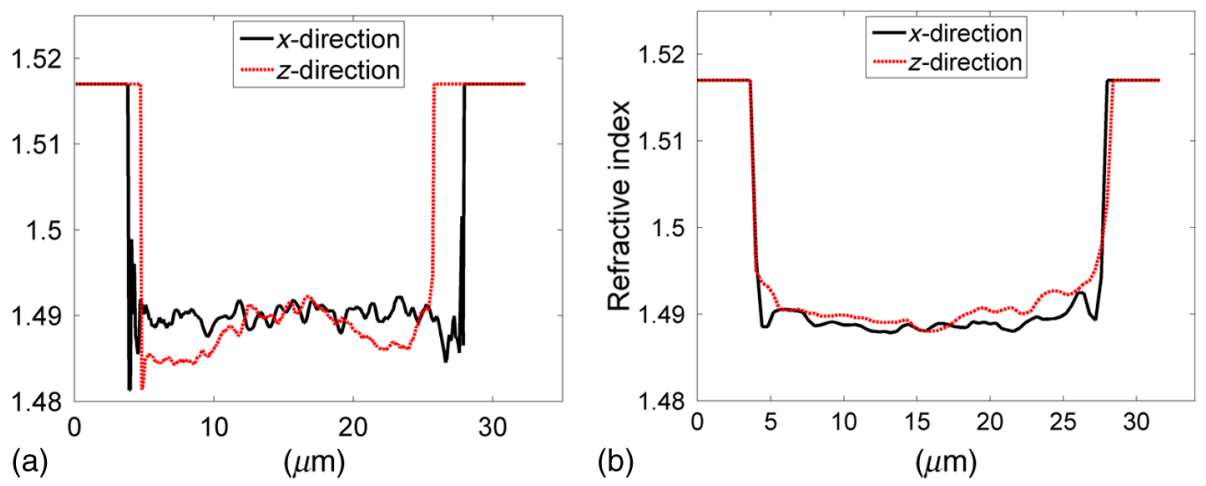

Fig. 10 The comparison of the microsphere 3-D refractive index distribution reconstruction profiles in two directions ( $X$ and $Z$ ) for (a) LSM scenario and (b) CSM scenario.

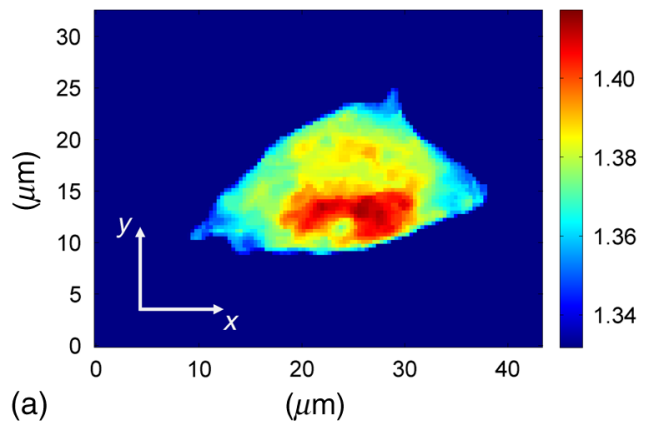

(a)

(c)

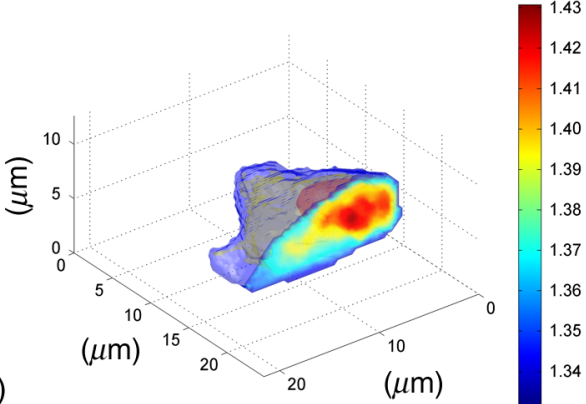

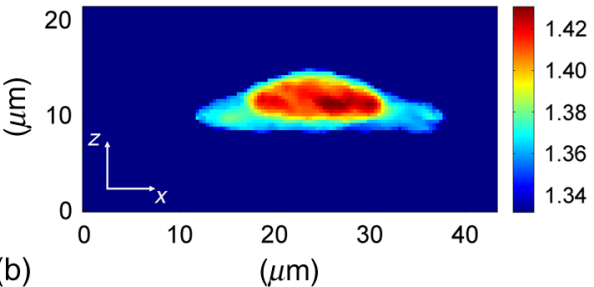

(b)

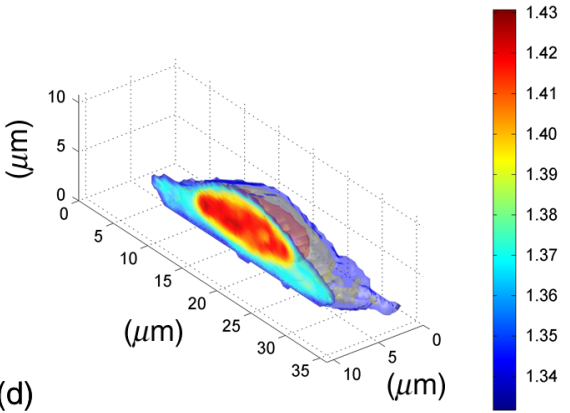

Fig. 11 Tomographic reconstruction of a single $\mathrm{C} 2 \mathrm{C} 12$ cell, measured using the CSM scenario and scaled to absolute refractive index values: (a) slice through the sample in the specimen plane $(X Y)$, (b) slice through the sample containing the optical axis $(X Z)$, (c) $Y Z$ slice in 3-D (Video 1, MPEG, 1.4 MB) [URL: http://dx.doi.org/10.1117/1.JBO.20.11.111216.1], and (d) $X Z$ slice in 3-D.

circular scanning measurement. In the case of the linear measurement, the inner structure of the microsphere was affected by the character of scanning and could be reduced if the linear scanning was performed in two directions. The reconstructed value of the refractive index in the center of the PMMA microsphere lies within the uncertainty usually estimated for limited-angle tomographic systems. ${ }^{32}$

\subsection{Tomographic Reconstruction of a Biological Sample}

In this section, we present the second step of the experiment, in which fixed adherent cells from the $\mathrm{C} 2 \mathrm{C} 12$ line were measured. Even though we did not measure living biological samples, our technique is equally suitable for such objects. The result of the measurement is shown in Fig. 11. In the reconstruction slices presented in Fig. 11(b), the region of highest refractive index values can be identified as the nucleus of the $\mathrm{C} 2 \mathrm{C} 12$ cell, and the nuclear envelope is visible. The regions of higher refractive index in the nucleus are also visible, which could correspond to the nucleoli. We find our result to be in agreement with DHMbased studies on the refractive index of the intracellular structures of adherent cells. ${ }^{59}$

The reconstructed cell example is artifact free and does not exhibit any significant error at the borders, which suggests that the algorithm performs better for nonpiecewise constant objects than the piecewise constant calibration object. However, to fully characterize the reconstruction quality and accuracy, a welldefined 3-D phase phantom would be very useful.

\section{Conclusion}

In this paper, new approaches to the projection generation in the holographic tomography measurement system and to the reconstruction using a limited set of projections were demonstrated. Placing an SLM in the tomographic microscope allows 
a vibration-free and robust operation. The optically controlled illumination angle is a convenient solution, due to the illumination scenarios it enables, which could be adjusted according to the measured object type. In addition, the SLM allows wavefront correction. This is an interesting feature, because although it is possible to numerically process the obtained phase images using propagation techniques or numerical aberration correction, the illuminating wavefront correction is not possible in this way. In the future, the performance of the wavefront correction should be verified and thoroughly tested. The algorithm proposed in the paper was proved to enhance the quality of the limited-angle reconstruction and extends the number of object types that can be successfully reconstructed to nonpiecewise constant samples, such as biological objects, e.g., cancer cells. It should be noted that tomographic studies of a calibrated object $^{32}$ usually do not focus on the quality of the slices at planes containing the optical axis, e.g., $X Z$ and $Y Z$ planes, since it is much easier to supplement the missing region of data in the Fourier domain in $k_{x}$ and $k_{y}$ directions than in $k_{z}{ }^{38}$ Improving the reconstruction in the direction of the optical axis while maintaining the correct reconstruction in the perpendicular direction was the main challenge of the reconstruction algorithm demonstrated in this paper; based on the calibrated object results, we claim this to be a success.

In the future, the method presented here should be implemented for the diffraction-based approach, which could lead to an improvement. Furthermore, in this paper only two basic measurement scenarios were tested. It would be beneficial to thoroughly analyze different possible sample illumination scenarios and determine the minimum number of projections and the optimum distribution of the scanning points in the focal plane of the illumination objective.

\section{Acknowledgments}

The research leading to the described results are realized within the program TEAM/2011-7/7 of Foundation for Polish Science, cofinanced from the European Funds of Regional Development. The authors would like to acknowledge the support from the grant of the Dean of Mechatronics Faculty, Warsaw University of Technology. Networking support was provided by the EXTREMA COST Action MP1207. We would like to thank Folkert Bleichrodt and Tristan van Leeuwen for providing the code for the Chambolle-Pock algorithm and Dr. Tytus Bernaś and Natalia Nowak from Laboratory of Imaging Tissue Structure and Function, Nencki Institute of Experimental Biology for providing biological samples.

\section{References}

1. T. Kim et al., "Breakthroughs in photonics 2013: quantitative phase imaging: metrology meets biology," IEEE Photonics J. 6, 1-9 (2014).

2. K. Lee et al., "Quantitative phase imaging techniques for the study of cell pathophysiology: from principles to applications," Sensors (Basel) 13, 4170-4191 (2013).

3. O. J. Pletjushkina et al., "Induction of cortical oscillations in spreading cells by depolymerization of microtubules," Cell Motil. Cytoskeleton 48, 235-244 (2001).

4. P. Pomorski et al., "How adhesion, migration, and cytoplasmic calcium transients influence interleukin-1 $\beta$ mRNA stabilization in human monocytes," Cell Motil. Cytoskeleton 57, 143-157 (2004).

5. P. Roy et al., "Microscope-based techniques to study cell adhesion and migration," Nat. Cell Biol. 4(4), E91-E96 (2002).
6. J. C. Petruccelli, L. Tian, and G. Barbastathis, "The transport of intensity equation for optical path length recovery using partially coherent illumination," Opt. Express 21, 14430-14441 (2013).

7. L. Waller et al., "Phase from chromatic aberrations," Opt. Express 18(22), 22817-22825 (2010).

8. L. Waller, L. Tian, and G. Barbastathis, "Transport of intensity phaseamplitude imaging with higher order intensity derivatives," Opt. Express 18, 12552-12561 (2010).

9. J. Martinez-Carranza, K. Falaggis, and T. Kozacki, "Optimum plane selection for transport-of-intensity-equation-based solvers," Appl. Opt. 53(50), 7050-7058 (2014).

10. J. Marrison et al., "Ptychography-a label free, high-contrast imaging technique for live cells using quantitative phase information," Sci. Rep. 3, 2369 (2013).

11. L. Tian et al., "Multiplexed coded illumination for Fourier ptychography with an LED array microscope," Biomed. Opt. Express 5, 23762389 (2014).

12. X. Ou et al., "High numerical aperture Fourier ptychography: principle, implementation and characterization," Opt. Express 23(3), 3472 (2015).

13. L. Tian and L. Waller, " $3 \mathrm{D}$ intensity and phase imaging from light field measurements in an LED array microscope," Optica 2, 104-111 (2015).

14. Z. Wang et al., "Spatial light interference microscopy (SLIM)," Opt. Express 19(2), 1016-1026 (2011).

15. M. Mir et al., "Visualizing Escherichia coli sub-cellular structure using sparse deconvolution spatial light interference tomography," PLoS One 7(6), e38916 (2012).

16. T. Kim et al., "White-light diffraction tomography of unlabelled live cells," Nat. Photonics 8, 256-263 (2014).

17. K. Alm et al., "Cells and holograms-holograms and digital holographic microscopy as a tool to study the morphology of living cells," in Holography-Basic Principles and Contemporary Applications, In Tech, Rijeka, HR (2013).

18. K. Kim et al., "Diffraction optical tomography using a quantitative phase imaging unit," Opt. Lett. 39(24), 6935-6938 (2014).

19. K. Lee and Y. Park, "Quantitative phase imaging unit," Opt. Lett. 39, 3630-3633 (2014).

20. G. G. Levin et al., "Shearing interference microscopy for tomography of living cells," Proc. SPIE 9536, 95360G (2015).

21. K. Kim et al., "High-resolution three-dimensional imaging of red blood cells parasitized by Plasmodium falciparum and in situ hemozoin crystals using optical diffraction tomography," J. Biomed. Opt. 19, 011005 (2014).

22. A. Kak and M. Slaney, Principles of Computerized Tomographic Imaging, IEEE Press, New York (1988).

23. Y. Kim et al., "Profiling individual human red blood cells using common-path diffraction optical tomography," Sci. Rep. 4, 6659 (2014).

24. V. Lauer, "New approach to optical diffraction tomography yielding a vector equation of diffraction tomography and a novel tomographic microscope," J. Microsc. 205, 165-176 (2002).

25. M. Debailleul et al., "Holographic microscopy and diffractive microtomography of transparent samples," Meas. Sci. Technol. 19, 074009 (2008).

26. A. Kus et al., "Limited-angle hybrid diffraction tomography for biological samples," Proc. SPIE 9132, 913200 (2014).

27. A. Barty et al., "Quantitative phase tomography," Opt. Commun. 175, 329 (2000).

28. B. Simon et al., "High-resolution tomographic diffractive microscopy of biological samples," J. Biophotonics 3, 462-467 (2010).

29. M. Debailleul et al., "Diffractive microscopy of transparent inorganic and biological samples," Opt. Lett. 34, 79-81 (2009).

30. W. Choi et al., "Tomographic phase microscopy," Nat. Methods 4, 717719 (2007).

31. C. Fang-Yen et al., "Video-rate tomographic phase microscopy," J. Biomed. Opt. 16, 011005 (2011).

32. Y. Sung et al., "Optical diffraction tomography for high resolution live cell imaging," Opt. Express 17(1), 266 (2009).

33. P. Xiu et al., "Controllable tomography phase microscopy," Opt. Lasers Eng. 66, 301-306 (2015).

34. M. Debailleul et al., "High-resolution three-dimensional tomographic diffractive microscopy of transparent inorganic and biological samples," Opt. Lett. 34(1), 79-81 (2009). 
35. Y. Cotte et al., "Marker-free phase nanoscopy," Nat. Photonics 7, 113117 (2013).

36. R. Chamgoulov, P. Lane, and C. MacAulay, "Optical computed-tomography microscope using digital spatial light modulation," Proc. SPIE 5324, 182-190 (2004).

37. W. Krauze, A. Kus, and M. Kujawinska, "Limited-angle hybrid optical diffraction tomography system with total-variation-minimization-based reconstruction," Opt. Eng. 54, 054104 (2015).

38. J. Lim et al., "Comparative study of iterative reconstruction algorithms for missing cone problems in optical diffraction tomography," Opt. Express 23(13), 16933 (2015).

39. B. Rappaz et al., "Digital holographic microscopy: a quantitative labelfree microscopy technique for phenotypic screening," Comb. Chem. High Throughput Screening 17, 80-88 (2014).

40. S. Sinzinger and J. Jahns, Microoptics, p. 168, Wiley-VCH, Weinheim, DE (2003).

41. J. Arines et al., "Measurement and compensation of optical aberrations using a single spatial light modulator," Opt. Express 15(23), 1528715292 (2007).

42. J. Zhang et al., "Wave front transformation and correction by using SLM," Proc. SPIE 6711, 67110C (2007).

43. O. Azucena et al., "Implementation of adaptive optics in fluorescent microscopy using wavefront sensing and correction," Proc. SPIE 7595, 75950I (2010).

44. J. Kacperski and M. Kujawinska, "Phase only SLM as a reference element in Twyman-Green laser interferometer for MEMS measurement," Proc. SPIE 6616, 66163E (2007).

45. F. Zernike, "Beugungstheorie des Schneidenverfahrens und Seiner Verbesserten Form, der Phasenkontrastmethode," Physica 1(8), 689704 (1934)

46. V. N. Mahajan and G.-M. Dai, "Orthonormal polynomials in wavefront analysis: analytical solution,” J. Opt. Soc. Am. A 24(9), 2994-3016 (2007).

47. M. Trusiak, M. Wielgus, and K. Patorski, "Advanced processing of optical fringe patterns by automated selective reconstruction and enhanced fast empirical mode decomposition," Opt. Laser Eng. 52, 230-240 (2014).

48. B. Bayraktar et al., "A numerical recipe for accurate image reconstruction from discrete orthogonal moments," Pattern Recognit. 40(2), 659-669 (2007).

49. P. C. Hansen, Discrete Inverse Problems: Insight and Algorithms, SIAM-Society for Industrial and Applied Mathematics, Pennsylvania, PA (2010).

50. D. Verhoeven, "Limited-data computed tomography algorithms for the physical sciences," Appl. Opt. 32(20), 3736-3754 (1993).

51. M. Ertas et al., "Digital breast tomosynthesis image reconstruction using 2D and 3D total variation minimization," Biomed. Eng. Online 12, 112 (2013).

52. M. Burger et al., "Total variation regularisation in measurement and image space for PET reconstruction," Numer. Anal. Inverse Problems 30(10), 1-30 (2014).
53. X. Jin et al., "Anisotropic total variation for limited-angle CT reconstruction," in IEEE Nuclear Science Symp. \& Medical Imaging Conf., pp. 2232-2238 (2010).

54. D. Chen et al., "The properties of SIRT, TVM, and DART for 3D imaging of tubular domains in nanocomposite thin-films and sections," Ultramicroscopy 147, 137-148 (2014).

55. A. Chambolle and T. Pock, "A first-order primal-dual algorithm for convex problems with applications to imaging," J. Math. Imaging Vision 40(1) 120-145 (2011).

56. A. Andersen, "Simultaneous algebraic reconstruction technique (SART): a superior implementation of the ART algorithm," Ultrasonic Imaging 6(1), 81-94 (1984).

57. W. J. Palenstijn, K. J. Batenburg, and J. Sijbers, "Performance improvements for iterative electron tomography reconstruction using graphics processing units (GPUs)," J. Struct. Biol. 176(2), 250-253 (2011).

58. D. Yaffe and O. Saxel, "Serial passaging and differentiation of myogenic cells isolated from dystrophic mouse muscle," Nature 270(5639), 725-727 (1977).

59. S. Przibilla, "Sensing dynamic cytoplasm refractive index changes of adherent cells with quantitative phase microscopy using incorporated microspheres as optical probes," J. Biomed. Opt. 17, 097001 (2012).

Arkadiusz Kuś is a PhD student at the Warsaw University of Technology. He received his MSc Eng degree in automation and robotics with specialization in photonics engineering from Warsaw University of Technology, Faculty of Mechatronics, in 2011. Apart from his scientific career, he also worked for the company PZO Microscopes and Optical Devices, Ltd., located in Warsaw, Poland. His current research interests include optical diffraction tomography, holography, and optical design.

Wojciech Krauze graduated from Warsaw University of Technology in 2013, where he studied photonics engineering in the Mechatronics Department and where he received his MSc degree. Currently, he is studying his PhD course at the Warsaw University of Technology. His main research tasks include developing algorithms for limited-angle tomography and new noninvasive methods for analysis of biological samples.

Małgorzata Kujawińska is a fellow of SPIE, a full professor of applied optics at the Warsaw University of Technology, and a head of the Photonics Engineering Division at the Institute of Micromechanics and Photonics. She is an expert in full-field optical metrology, hybrid optonumerical methods in mechanics, image processing, automatic data analysis, and design of novel photonics systems. Since 1980 she has been involved in optical metrology topics including development of interferometric, holographic, grating interferometry, diffraction tomography, digital image correlation, and structured light based methods. She is the author of one monograph, several book chapters, and more than 200 papers in international scientific journals. 